CRECIMIENTO ECONÓMICO Y DESALINEACIÓN DEL TIPO DE CAMBIO REAL EN LA REPÚBLICA DOMINICANA: ¿HAY ALGUNA RELACIÓN?

Economic growth and the real exchange rate misalignments in the Dominican Republic: Is there a link?

\title{
Alexis Cruz-Rodríguez*
}

Resumen: El objetivo de este artículo es examinar el efecto de la desalineación del tipo de cambio real sobre el crecimiento económico de la República Dominicana. Para llevar a cabo el análisis se construyeron seis medidas de desalineación. Los resultados empíricos indican que los coeficientes para las distintas medidas de desalineación del TCR, son negativos en todos los modelos estimados y estadísticamente significativos en la mayoría, lo que significa que un tipo de cambio real más depreciado tiene un efecto negativo en el crecimiento de largo plazo.

Palabras clave: Tipo de cambio real, desalineación cambiaria, crecimiento económico, República Dominicana.

JEL clasificación: F31, F41 O11

\footnotetext{
Pontificia Universidad Católica Madre y Maestra. Correo electrónico: alexiscruz@ pucmm.edu.do

Se agradecen los comentarios de Amelia U. Santos-Paulino, José R. Sánchez-Fung y de los asistentes al taller INTEC-Universidad de Londres The Dominican Republic's Economy celebrado en el Instituto de Estudios Latinoamericanos, Universidad de Londres, Reino Unido, el 7 de noviembre de 2014. Se agradecen también los comentarios de los asistentes a la Semana de Investigación de la Pontificia Universidad Católica Madre y Maestra, en marzo 2015. Todos los errores son de única responsabilidad del autor.
} 
Abstract: The aim of this article is to examine the effect of the real exchange rate misalignment on the economic growth in the Dominican Republic. The paper constructs six measures of exchange rate misalignment. The empirical results indicate that the coefficients for the different measures of RER misalignment are negative in all estimated models and statistically significant in most, which means that a real exchange rate more depreciated has a negative effect on growth long term.

Keywords: Real exchange rate, exchange rate misalignment, economic growth, Dominican Republic.

JEL classification: F31, F41, O11

\section{Introducción}

Los factores determinantes del crecimiento económico han sido ampliamente estudiados por la literatura especializada, tanto teórica como empírica. Sin embargo, tanto en los trabajos de crecimiento económico neoclásico (Solow, 1956) como en los de crecimiento endógeno (Romer, 1986; Lucas, 1988) el tipo de cambio real (TCR) no ha sido una variable de primer orden para explicar la dinámica del crecimiento. No obstante, en los últimos diez años el tipo de cambio real ha cobrado relevancia por dos razones, primero, como resultado de trabajos empíricos que han detectado la existencia de una correlación positiva entre el crecimiento económico y la desalineación del TCR (medida de formas alternativas), y segundo, por la apreciación del tipo de cambio real experimentada por varias economías, tanto en desarrollo como desarrolladas, dado que el dólar estadounidense ha mostrado una tendencia a la 
depreciación debido al déficit fiscal y de cuenta corriente mostrado por la economía norteamericana. Pero la idea de una relación del TCR y el crecimiento económico se remonta a los años 80 con la llamada estrategia de desarrollo "hacia afuera" llevada a cabo por algunas economías en desarrollo, la cual sostenía la importancia de contar con un tipo de cambio real más "competitivo" para incentivar las inversiones orientadas a la exportación y el crecimiento económico. Pero un tipo de cambio real más "competitivo" involucra un proceso de devaluación o depreciación del tipo de cambio nominal. La lógica era la siguiente, un tipo de cambio real más depreciado (subvaluado) conduce a los factores de producción hacia el sector transable que tienden a tener altas tasas de crecimiento. Sin embargo, un tipo de cambio nominal depreciado o devaluado podría desencadenar un proceso inflacionario. En ese sentido, el Consenso de Washington sostuvo que los desalineamientos cambiarios eran nocivos para el crecimiento económico.

Es importante destacar que el concepto de desalineación del tipo de cambio real describe una situación en la que la tasa de cambio real efectiva de un país se desvía de su nivel de largo plazo o nivel de equilibrio sostenible. Por tanto, un tipo de cambio real estaría en equilibrio cuando promueva una tasa de crecimiento de las exportaciones que permita mantener el déficit en cuenta corriente en un nivel que pueda ser financiado de manera sostenida. Si el tipo de cambio es más competitivo que eso, entonces podrían producirse presiones inflacionarias innecesarias que reduzcan los recursos disponibles para la inversión doméstica y, a su vez, limiten el crecimiento del producto potencial. En consecuencia, un tipo de cambio real por encima de su valor de equilibrio no necesariamente conduce a un mayor crecimiento económico (Williamson, 1990). Desde este punto de vista, el desarrollo económico exitoso resulta en una apreciación de la tasa de cambio real y en un mejoramiento del nivel de vida, mientras que el fracaso se traduce en una fuerte depreciación de la moneda de los países. 
El objetivo de este trabajo es abordar la relación, si es que existe, entre la desalineación del TCR y el crecimiento económico exhibido por la República Dominicana. Para ello, se efectúan diferentes regresiones de medidas alternativas de desalineamiento del TCR con el crecimiento económico. El resto de este artículo está organizado de la siguiente manera: la sección 2 presenta una revisión de la evidencia empírica internacional respecto a la relación entre crecimiento y tipo de cambio real. En la sección 3 se discuten distintas formas en las que es posible definir el tipo de cambio real de equilibrio (TCRE) en la práctica y se calcula el desalineamiento a partir de distintas nociones de equilibrio. La sección 4 muestra los aspectos metodológicos del modelo utilizado para evaluar el impacto del desalineamiento sobre el crecimiento, y la fuente de datos utilizados. La sección 5 muestra los resultados empíricos. Finalmente, en la sección 6 se presentan algunas conclusiones.

\section{Revisión de la literatura}

La literatura sobre el equilibrio del tipo de cambio real se remonta a la década de 1960 con los trabajos de Balassa (1964), pero su relación con el crecimiento económico se inicia a fines de los años ochenta. Uno de los primeros estudios sobre la desalineación y el crecimiento del tipo de cambio es el desarrollado por Edwards (1989). Dicho autor investiga la relación entre el tipo de cambio real y el crecimiento, y en una de las conclusiones principales señala que las desalineaciones de los tipos de cambio reales están asociadas con las distorsiones de precios relativos en los sectores transables y no transables, lo que genera una asignación no óptima de los recursos entre los diferentes sectores de la economía, por tanto, la desalineación tiene un impacto negativo en el crecimiento. De igual forma, Cottani, Cavallo y Khan (1990) examinan el comportamiento del TCR y el desempeño económico de veinticuatro economías en desarrollo durante el período 1960-1983. Sus resultados muestran una relación negativa entre la desalineación del TCR y el crecimiento. Asimismo, Razin y Collins (1997), construyen 
un panel para 93 países en el período 1975-1993. Sus resultados sugieren que solamente muy altas sobrevaluaciones o apreciaciones del TCR están asociadas con el bajo crecimiento, mientras que moderadas o altas (pero no muy altas) subvaluaciones o depreciaciones están asociadas con un rápido crecimiento económico. Adicionalmente, estos autores muestran la no linealidad entre el TCR y el crecimiento.

Por su parte, Domac y Shabsigh (1999) investigan la relación entre la desalineación del tipo de cambio real y el crecimiento económico para Egipto, Jordania, Marruecos y Túnez en el período 19701996. Siguiendo a Cottani, Cavallo y Khan (1990) los autores calculan la desalineación como variaciones del tipo de cambio real con respecto al promedio del período bajo estudio. Sus resultados muestran un efecto negativo de la desalineación del tipo de cambio real sobre el crecimiento económico para las economías consideradas. Mientras Ito, Isard y Symansky (1999) examinan la relación entre la tasa de crecimiento económico y los cambios en el tipo de cambio real en los países miembros del Acuerdo de Cooperación Económica del Asia-Pacífico (APEC, por sus siglas en inglés), en el período 1973-1995. Sus resultados muestran que algunos países tienen una relación positiva entre depreciación del tipo de cambio real y el crecimiento económico, pero otros muestran una relación negativa. Asimismo, Aguirre y Calderón (2006) también encontraron que los desalineamientos del TCR reducen el crecimiento pero de forma no lineal. Estos autores calcularon el desalineamiento en el TCR como desviaciones del TCR efectivo de su equilibrio. La medida de desalineamiento del tipo de cambio fue calculada sobre la base de los residuos de una regresión FEER (Fundamental Equilibrium Exchange Rate) y utilizaron análisis de cointegración y paneles dinámicos para 60 países en el período 1965-2003. La evidencia empírica mostrada por los autores, sugiere que el efecto de la desalineación del TCR sobre el crecimiento no es lineal, lo que significa que cuando la depreciación del tipo de cambio real es demasiado alta, el impacto sobre el crecimiento es 
negativo, pero cuando es pequeña o moderada pueden incrementar el crecimiento. Además, dichos autores consideran que fuertes apreciaciones del TCR también tendrían efectos negativos en el crecimiento. Por el contrario, Gala y Lucinda (2006) desarrollaron un análisis de datos de panel dinámico para un conjunto de 58 países desde 1960 hasta 1999. La principal evidencia empírica apoya el argumento de que un depreciado (apreciado) tipo de cambio real está asociado a mayores (menores) tasas de crecimiento.

En otro orden, Eichengreen (2008) presenta una breve reseña histórica sobre la relación entre el tipo de cambio real y el crecimiento de la actividad económica, centrando la atención en los posibles canales por los que el tipo de cambio real podría tener un impacto en el crecimiento económico a largo plazo. ${ }^{1}$ El autor argumenta a favor de un tipo de cambio real más depreciado para fomentar las exportaciones y el crecimiento económico, siempre y cuando dicha depreciación no esté asociada con una mayor volatilidad del tipo de cambio porque de ocurrir, el impacto sería negativo. De igual forma, Gala (2008), utilizando datos de panel para 58 economías en desarrollo, en el período 1960-1999, encuentra que la subvaluación del tipo de cambio real está asociada con el incremento en el ingreso per cápita de dichos países. De forma similar, Rodrick (2008) examinó la relación del tipo de cambio real con el crecimiento económico, usando diferentes técnicas de estimación, para una muestra de 188 países en el período 1950-2004. Sus resultados sugieren que un tipo de cambio real subvaluado (depreciado) estimula el crecimiento económico, particularmente en economía en desarrollo. El autor desarrolla un índice para medir el grado de subvaluación del tipo de cambio real, ajustado por el efecto Balassa-Samuelson utilizando el PIB real per cápita.

1 Para una revisión de la literatura sobre la desalineación del tipo de cambio se recomienda revisar a Edwards y Savastano (1999). 
Por su parte, Berg y Miao (2010) realizan una investigación empírica sobre el crecimiento y la desalineación del tipo de cambio real, con el fin de comparar los resultados de Rodrik (2008) con la visión del Consenso de Washington. Sus resultados sugieren que ambos puntos de vista son equivalentes econométricamente. Los resultados empíricos mostrados por estos autores apoyan los de Rodrik (2008) en el sentido de que la subvaluación del TCR ayuda a fomentar el crecimiento a largo plazo, mientras que la sobrevaluación tiene el efecto contrario. No obstante, dicho resultado no es coherente con la opinión del Consenso de Washington. Asimismo, Abida (2011), siguiendo a Berg y Miao (2010), investiga el impacto de la desalineación del tipo de cambio real en Túnez, Argelia y Marruecos en el período 1980-2008. Sus resultados indican que un tipo de cambio real más depreciado, con respecto a su nivel de equilibrio, contribuye positivamente al crecimiento económico en el largo plazo. Similarmente, McLoed y Mileva (2011), utilizando datos de panel para 58 países en desarrollo, en el período 1975-2004, encuentran una relación positiva entre depreciación del tipo de cambio real y el crecimiento. De esta forma, Razmi, Rappetti y Skott (2012), usando un dato de panel dinámico (método generalizado de momento) para una muestra de 153 países en el período 1960-2004, encuentran una relación positiva entre el tipo de cambio real depreciado y el crecimiento de la inversión, pero particularmente en economías en desarrollo. Similarmente, Rapetti, Skott y Razmi (2012) usando datos de panel para una muestra de 181 países en el período 1950-2004, encontraron que la depreciación del tipo de cambio real tiene un efecto positivo en el crecimiento económico de los países en desarrollo. En ese sentido, Mbaye (2012), utilizando datos de panel para 72 países en el período 1970-2008, encuentra una relación positiva entre la subvaluación del TCR y el crecimiento económico, pero esta relación es posible por la vía de un incremento de productividad. De igual forma, Vieira y MacDonald (2012), investigan el papel jugado por la desalineación del tipo de cambio real en el crecimiento económico de largo plazo, para una muestra de 90 países en el período 1980-2004. Sus 
resultados sugieren que un TCR más depreciado contribuye positivamente con el crecimiento económico de largo plazo. Sin embargo, cuando la depreciación es muy alta, la misma tiene un efecto negativo en el crecimiento de largo plazo. Además, estos autores no encuentran evidencia de no linearidad entre la desalineación del TCR y el crecimiento económico. De igual forma, Beréau, López Villavicencio y Mignon (2012) analizan la relación entre la desalineación del TCR y el crecimiento. Estos autores utilizan una muestra de 32 países en el período 1980-2007 y encuentran una relación positiva entre la depreciación y el crecimiento económico.

Por su parte, Henrique Vaz y Baer (2014) examinan la relación entre la desalineación del TCR y el sector manufacturero, para una muestra de 39 países latinoamericanos en el período 1995-2008, encontrando una relación positiva entre ellos. De igual forma, Debowicz y Saeed (2014), utilizando un modelo de Vectores Autorregresivos (VAR), encuentran una relación positiva entre la depreciación cambiaria y el crecimiento económico de Pakistán, en el período 1982-2010. Por el contrario, Bussiere, López y Tille (2014) analizan el efecto de la apreciación del TCR en el crecimiento para una muestra de 68 economías (30 avanzadas y 38 emergentes), en el período 1960-2011. Sus resultados sugieren que, primero, la apreciación del TCR está asociada con bajo crecimiento solo en economías avanzadas y en períodos normales (ausencia de turbulencias), y segundo, tanto las economías avanzadas como las emergentes presentan crecimiento económico con apreciación del TCR cuando al mismo tiempo se produce un incremento de la productividad. De igual forma, Griffin (2015) analiza la relación del TCR y el crecimiento del sector manufacturero en Colombia, en el período 2000-2012. Este autor no encuentra una evidencia fuerte de que la apreciación real afecte negativamente la rentabilidad de las empresas manufactureras. Por el contrario, sus resultados sugieren que la apreciación real puede haber aumentado la rentabilidad de las empresas mediante la reducción del costo de insumos importados. 
En el caso de la economía dominicana los trabajos sobre crecimiento económico no han considerado el papel del TCR como determinante del mismo (Montiel, 2006; Jaramillo y Sancak, 2007; Fanelli y Guzmán, 2008; y Pozo, Sánchez-Fung y Santos-Paulino, 2011, entre otros). No obstante, Fanelli y Guzmán (2008) reconocen el papel del TCR en el desempeño del sector transable, a raíz de los cambios estructurales de principios de la década del noventa.

\section{Definiciones alternativas del tipo de cambio real de equilibrio (TCRE) y medición del desalineamiento ${ }^{2}$}

En este trabajo se define desalineación del TCR como la desviación del tipo de cambio real actual con respecto a su nivel de equilibrio. Por tanto, la primera pregunta que surge al evaluar los efectos del tipo de cambio real sobre el crecimiento de la actividad económica es cuál va a ser el valor de equilibrio o de referencia con el cual vamos a comparar los datos observados y definir el grado de desalineamiento. Para realizar este trabajo, se utilizaron dos medidas diferentes de tipos de cambio real y seis medidas de desalineamiento. Primero, se utiliza un tipo de cambio real bilateral con respecto a Estados Unidos construido de la siguiente forma:

$$
\ln t c r_{I P C_{t}}=\ln \left(\frac{E_{t} \times P P I_{U S_{t}}}{I P C_{R D_{t}}}\right)
$$

donde $E$ es el tipo de cambio nominal con respecto al dólar, PPI es el índice de precios al productor de los Estados Unidos e IPC es el índice de precios al consumidor de la República Dominicana. Para generar una medida de desalineamiento a partir de la ecuación (1) se optó por dos alternativas. Primero, se optó por calcular la desviación porcentual del tipo de cambio real respecto a su media móvil de los últimos cinco años, esto reflejaría aproximadamente el equilibrio de paridad del poder adquisitivo (PPP, por sus siglas en inglés), y luego

2 Esta sección se basa en Cruz-Rodríguez (2015). 
se procedió a restar dicho promedio (considerado el tipo de cambio real de equilibrio) del dato observado. Formalmente tendríamos lo siguiente: $\ln$ Destcr $_{t}=\ln t c r_{t}-\ln t \hat{c} r_{t}$. Si se obtiene un valor mayor (menor) a cero significa que el TCR está depreciado (apreciado) con respecto al equilibrio, lo que significa que los bienes producidos domésticamente son relativamente más baratos (caros) en términos del dólar norteamericano. A este enfoque le llamaremos

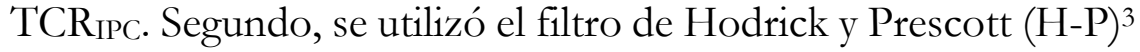
para obtener el tipo de cambio real de tendencia suavizada al que consideramos como de equilibrio, y luego se le restó al dato observado, como procedimos anteriormente. A este enfoque de desalineación del TCR le llamaremos TCR H-P. $_{\text {. }}$ Si se obtiene un valor mayor (menor) a cero significa que el TCR está depreciado (apreciado) con respecto al equilibrio.

La segunda medida de tipo de cambio real utilizada en este trabajo se basa en el enfoque de la paridad del poder adquisitivo ajustada por el efecto Balassa-Samuelson. ${ }^{4}$ Es decir, se utiliza un tipo de cambio real corregido por la paridad del poder de compra o adquisitivo (PPP). Este enfoque postula un tipo de cambio real de equilibrio invariante en el tiempo, al cual deberá converger el tipo de cambio observado (siempre que no se limite la posibilidad de hacer arbitraje y se cumplan una serie de supuestos). Si se incluye el sector no transable, la diferencia en productividades relativas

3 Hodrick y Prescott (1997) sugieren un valor para el parámetro de suavización $(\boldsymbol{\lambda})$ de 100 , para trabajar con datos anuales. Sin embargo, la literatura económica sugiere que la elección, un tanto arbitraria, del parámetro de suavización es una limitación de esta metodología. No obstante lo anterior, para datos anuales no existe consenso y la utilización del parámetro de suavización va desde $\lambda=10$ hasta $\lambda=400$. Sin embargo, Ravn y Uhlig (2001) demuestran empíricamente que el parámetro $\lambda$ propuesto por Hodrick y Prescott para datos anuales debe ser ajustado, aproximando las propiedades del filtro trimestral para otras frecuencias tras lo cual obtienen un $\lambda$ de 6.25 para series anuales. Dado el consenso con respecto al parámetro de suavización, las estimaciones realizadas en este trabajo utilizan el ajuste sugerido por Ravn y Uhlig para datos anuales (6.25).

${ }^{4} \mathrm{El}$ atractivo para utilizar esta teoría en la determinación del TCRE es su simplicidad y la intuición que está detrás: el arbitraje y la teoría de un solo precio. 
de dichos sectores de la economía doméstica y el resto del mundo afectará el nivel del TCRE de PPP (Balassa, 1964; Samuelson, 1964). ${ }^{5}$ De esta forma y siguiendo a Rodrick (2008), se construye una medida de tipo de cambio real definida a partir de la siguiente expresión:

$$
\ln t c r_{P p p_{t}}=\ln \left(\frac{E_{t}}{P P P_{t}}\right)
$$

donde $E$, al igual que en la ecuación anterior es el tipo de cambio nominal con respecto al dólar y la variable PPP es el factor de conversión para ajustar por poder adquisitivo entre distintas monedas. ${ }^{6}$ Cuando el tipo de cambio real se presente de acuerdo a la ecuación (2), números mayores a uno indican que el valor de la moneda es menor que lo que indica la $P P P$, es decir, la moneda está más depreciada de lo que indica la paridad del poder adquisitivo. Por tanto, esta es nuestra tercera medida de desalineación la cual llamaremos TCR ppr. Sin embargo, esta medida no toma en cuenta que en países con bajos niveles de ingreso per cápita los bienes y servicios no transables suelen ser más baratos (efecto Balassa-Samuelson), lo cual es una limitante. Por tanto, para construir una medida de desalineación primero se necesita corregir por dicho efecto, para ello seguimos la estrategia trazada por Rodrick (2008) y Berg y Miao (2010). Estos autores estiman una ecuación como la siguiente:

$$
\ln \operatorname{tcrppp}_{t}=\alpha+\beta \ln y_{t}+\varepsilon_{t}
$$

donde $y$ es el PIB per cápita anual y $\varepsilon$ es un término de error. Los resultados de la estimación de la ecuación (3) se presentan en la tabla N. ${ }^{\circ}$. El PIB per cápita tiene el signo correcto, lo que significa que está en línea con el efecto Balassa-Samuelson y es significativo

\footnotetext{
5 Para una revisión del efecto Balassa-Samuelson ver a Harberger (2003).

6 El tipo de cambio PPP en la ecuación (2) es un cociente de precios relativos, es decir, es el precio de una canasta de bienes medidos en moneda local entre países. Formalmente, la ecuación de $t c r=\frac{E P^{*}}{P}$ se puede re-escribir como $t c r=\frac{E}{\left(\frac{P}{P^{*}}\right)}$ que es equivalente a la ecuación (2).
} 
al 1\% (con un alto t-estadístico). Cuando el ingreso se incrementa en $10 \%$ el tipo de cambio real se aprecia en 10.9\%. Estos resultados son consistentes con los encontrados por Razmi, Rappetti y Skott (2012).

Tabla N. ${ }^{\circ} 1$

Resultados de la estimación del efecto Balassa-Samuelson

\begin{tabular}{l|c}
\hline \multicolumn{1}{c|}{ Variable } & TCR $_{\text {PPP }}$ \\
\hline Constante & $1.853^{*}$ \\
& $(0.333)$ \\
PIB per cápita & $-0.109^{*}$ \\
& $(0.033)$ \\
Prob (F-estadístico) & 0.002 \\
$\mathrm{R}^{2}$ & 0.152 \\
\hline
\end{tabular}

Fuente: Elaboración propia.

Nota: La variable dependiente es $\operatorname{lntcr}$ ppp. Los errores estándar están en paréntesis. Los símbolos *, \# y ^ representan un nivel de significancia de $1 \%, 5 \%$ y $10 \%$, respectivamente.

Una vez controlado el efecto Balassa-Samuelson, construimos el desalineamiento como resultado de la diferencia entre el tipo de cambio real predicho a partir de la regresión de la ecuación $(3)^{7}$ y el tipo de cambio observado (TCRPPP) de la ecuación (2). Si la diferencia entre ambos resulta positiva (negativa) se dice que el tipo de cambio real está depreciado (apreciado) respecto al equilibrio. A este enfoque de desalineación del TCR le llamaremos TCR B-S.

Finalmente, se utiliza un modelo de comportamiento BEER (Behavioral Equilibrum Exchange Rate). La idea es obtener un TCRE construido sobre la base de una estimación econométrica que relacione el tipo de cambio real observado con algunas variables consideradas como "fundamentales" o determinantes. Las variables

\footnotetext{
7 Se realizó una proyección dentro de muestra.
} 
o determinantes utilizados en esta regresión son las recomendadas por la literatura especializada ${ }^{8}$ : el efecto Balassa-Samuelson, el tamaño del sector público (consumo de gobierno como porcentaje del PIB), los términos de intercambio, el grado de apertura y la inversión como porcentaje del PIB. La ecuación a estimar es la siguiente:

$$
\text { ltcr }_{t}=\alpha+\beta \ln y_{t}+\gamma X_{t}+\mu_{t}
$$

donde Itcr es la variable dependiente que se construye como la ecuación (1) y (2), $y$ es el PIB per cápita anual, y la variable $X$ es un vector que incluye los determinantes señalados en el párrafo anterior. Los resultados de la estimación de la ecuación (4) se presentan en la tabla N. ${ }^{\circ} 2$. Los signos de las variables, así como su significancia estadística son acorde a las encontradas por otros investigadores. Una subida de los términos de intercambio, del gasto público y/o de la inversión tiende a apreciar el tipo de cambio real. Se destaca la consistencia en el valor estimado del coeficiente que captura el efecto Balassa-Samuelson (ver tabla N. ${ }^{\circ} 1$ del enfoque PPP).

El cálculo de la desalineación es similar al utilizado en el enfoque anterior, dado que será el resultado de la diferencia entre el tipo de cambio real predicho a partir de la regresión de la ecuación (4) y el tipo de cambio observado. Si la diferencia entre ambos resulta positiva (negativa) se dice que el tipo de cambio real está depreciado (apreciado) respecto al equilibrio. ${ }^{9}$ A estos dos enfoques de desalineación del TCR le llamaremos TCR respectivamente.

8 Sin embargo es importante destacar que no existe en la literatura especializada un consenso absoluto sobre el set de variables o fundamentales apropiados para este tipo de análisis.

9 Una limitante de esta metodología es que el desalineamiento recoge todos los errores de especificación y medición del modelo. Es decir, si el poder explicativo del modelo es muy bueno, por construcción, no existirá desalineamiento. En cambio, si el poder explicativo no es bueno habrá un alto desalineamiento con respecto a equilibrio. 
Tabla N. ${ }^{\circ} 2$

Resultados de la estimación del BEER

\begin{tabular}{l|c|c}
\hline \multicolumn{1}{c|}{ Variable } & LTCR & LTCRPPP \\
\hline Constante & $3.015^{*}$ & $0.958 \#$ \\
& $(12.130)$ & $(0.409)$ \\
PIB per cápita & $0.048 \#$ & -0.029 \\
& $(2.198)$ & $(0.036)$ \\
Consumo del gobierno & -0.281 & $-1.446^{\wedge}$ \\
& $(0.446)$ & $(0.729)$ \\
Términos de Intercambio & -0.037 & -0.074 \\
& $(0.092)$ & $(0.151)$ \\
Apertura & $0.790^{*}$ & $0.994^{*}$ \\
Inversión & $(0.095)$ & $(0.162)$ \\
& $-0.411^{*}$ & $-0.518^{*}$ \\
Prob (F-estadístico) & $(0.067)$ & $(0.108)$ \\
& 0.000 & 0.000 \\
\hline
\end{tabular}

Fuente: Elaboración propia.

Nota: Los errores estándar están en paréntesis. Los símbolos *, \# y ^ representan un nivel de significancia de $1 \%, 5 \%$ y $10 \%$, respectivamente.

\section{Metodología y datos}

Para evaluar la relación entre desalineamiento del tipo de cambio real y el crecimiento económico vamos a estimar tres ecuaciones. Primero, vamos a estimar una ecuación de la siguiente forma:

$$
\Delta y_{t}=\beta_{0}+\beta_{1} y_{t-1}+\delta_{0} \text { Destcr }_{t}+\eta_{t}
$$

donde $\Delta y_{t}$ es la tasa de crecimiento anual del PIB per cápita, medida en $\mathrm{RD} \$$ reales, $y_{t-1}$ significa el nivel del PIB per cápita es el período previo, es decir, el PIB per cápita inicial, Destcr es la desalineación 
del tipo de cambio real (medida bajo las formas alternativas definidas anteriormente) y $\eta$ es un término de error. Se espera que la variable PIB inicial tenga un signo negativo, dado que su efecto está relacionado con la convergencia condicional. A menor ingreso inicial mayor será la tasa de crecimiento $^{10}$.

La segunda ecuación a estimar incluye un set de determinantes del crecimiento identificados en la literatura y el rezago de la desalineación. De esta forma, se estima la siguiente ecuación:

$\Delta y_{t}=\beta_{0}+\beta_{1} y_{t}+\delta_{0}$ Destcr $_{t}+\delta_{1}$ Destcr $_{t-1}+\rho X_{t}+\eta_{t}$

donde $\Delta y_{t}$ es la tasa de crecimiento del PIB per cápita y está medida en $\mathrm{RD} \$$ reales, $X$ es un vector que incluye los determinantes adicionales del crecimiento acorde con la literatura, Destcr es la desalineación del tipo de cambio real con respecto a su valor de equilibrio (medida bajo las formas alternativas definidas anteriormente), y $\eta$ es un término de error. Los determinantes incluidos en la ecuación son escolaridad o educación, el grado de apertura de la economía, la inflación, la población, el consumo del gobierno como porcentaje del PIB, la inversión como porcentaje del PIB y los términos de intercambios. Con respecto a la educación, dicha variable está medida como el promedio de años de escolaridad para personas mayores a 15 años. Esta variable es una proxy del stock de capital humano y se espera un signo positivo dado que los países con más capital humano tienden a crecer más rápido. El grado de apertura de la economía está medido como el ratio de la suma de las exportaciones y las importaciones sobre el PIB. Para esta variable se espera un signo positivo. La inflación está medida como la variación anual del Índice de Precios al Consumidor (IPC) y se incluye como una proxy de pérdida de estabilidad de precios o de estabilidad monetaria, por tanto, se espera un signo negativo para dicha variable. En cuanto a la población, el

10 Véase a Barro y Sala-i-Martin (1990).

Ciencia y Sociedad 2015; 40(3): 571-605 585 
signo esperado para esta variable es ambiguo, por un lado, países con alto crecimiento de la población tienden a tener altas tasas de crecimiento (si la misma afecta positivamente la función de producción), pero altas tasas de crecimiento de la población puede estar asociado con bajas tasas de crecimiento del PIB per cápita. Con relación al tamaño del gobierno, medido como el ratio consumo de gobierno a PIB, existe un antiguo debate en la política económica dentro de los países en desarrollo que se refiere al efecto que ejerce el tamaño del gobierno en el crecimiento económico. Algunos autores, basados en los modelos de crecimiento endógeno, esperan un signo negativo dado que dicha variable se considera también como una proxy de estabilidad fiscal, es decir, un incremento en el consumo de gobierno aumentará el volumen de tributación distorsionador y, por tanto, se reducirá el crecimiento. Además, un canal adicional mediante el cual el consumo del gobierno puede afectar negativamente el crecimiento es el desplazamiento de la inversión del sector privado (efecto expulsión). ${ }^{11}$ En cuanto a la inversión como porcentaje del PIB, se espera un signo positivo debido a que la acumulación de capital conduce a un mayor crecimiento del PIB real per cápita. ${ }^{2}$ Por su parte, los términos de intercambio son el resultado del ratio de precios entre exportación e importación y se espera un signo positivo para dicha variable.

En orden de investigar si la sobrevaluación o subvaluación tienen diferente impacto sobre el crecimiento, se estima una tercera ecuación con la siguiente especificación:

$$
\Delta y_{t}=\beta_{0}+\beta_{1} y_{t}+\delta_{0} \text { Dummy }_{t}+\rho X_{t}+\eta_{t}
$$

11 No obstante, se reconoce que el gasto gubernamental también puede ser complementario de la inversión del sector privado.

12 Sin embargo, en un equilibrio de estado estable en el largo plazo, la tasa de acumulación de capital es igual al producto, por ello, esta variable por lo regular no aparece en las ecuaciones de crecimiento (Barro, 1991). Pero se incluye aquí porque no consideramos que el modelo utilizado pueda interpretarse como de estado estable (Edwards, 1992). 
donde $\Delta y_{t}$ es la tasa de crecimiento del PIB per cápita y está medida en $\mathrm{RD} \$$ reales, $X$ es un vector que incluye los determinantes mencionados anteriormente, Dummy es una variable ficticia que asume el valor de uno cuando la desalineación implica una depreciación del TCR, y de cero en otros casos, y $\eta$ es un término de error. Adicionalmente, se re-estima la ecuación (7) modificando la variable Dummy que asumirá el valor de uno cuando la desalineación implica una apreciación del TCR, y de cero en otros casos.

Con respecto a los datos utilizados en este trabajo, los mismos tienen una frecuencia anual y abarcan el período 1950-2013. Las fuentes consultadas para la base de datos fueron el Banco Mundial, el Fondo Monetario Internacional, el Banco Central de la República Dominicana y la Penn World Table.

\section{Resultados}

Esta sección está dividida en tres partes. Primero se presentan los resultados de la estimación de la ecuación (5) y luego los resultados de los determinantes del crecimiento económico dados por las ecuaciones (6) y (7). En los tres casos se presentan los resultados utilizando las distintas definiciones de desalineación del tipo de cambio real, pero en la estimación de la ecuación (5) empleamos índices en niveles del TCR como sustitutos de la desalineación. Con ello, medimos la sensibilidad de los resultados a enfoques alternativos respecto al equilibrio, lo cual es uno de los test de robustez más relevante.

De esta forma, en la tabla N. ${ }^{\circ} 3$ pueden observarse los resultados de la estimación de la relación entre el desalineamiento de TCR, obtenido por los distintos enfoques explicados oportunamente en este trabajo, y el crecimiento económico de la República Dominicana (columnas 1, 2, 3, 4, 5 y 6). Asimismo, se corrieron regresiones empleando los índices en niveles del TCR (columnas 7 y 8). Al igual que Rodrik (2008) y Razmi et al. (2012) suponemos 
que la desalineación está dominada por una subvaluación o depreciación del tipo de cambio real. Por tanto, podemos referirnos indistintamente a subvaluación o desalineación.

Tabla N. ${ }^{\circ} 3$

Resultados relación de la desalineación del TCR y el crecimiento

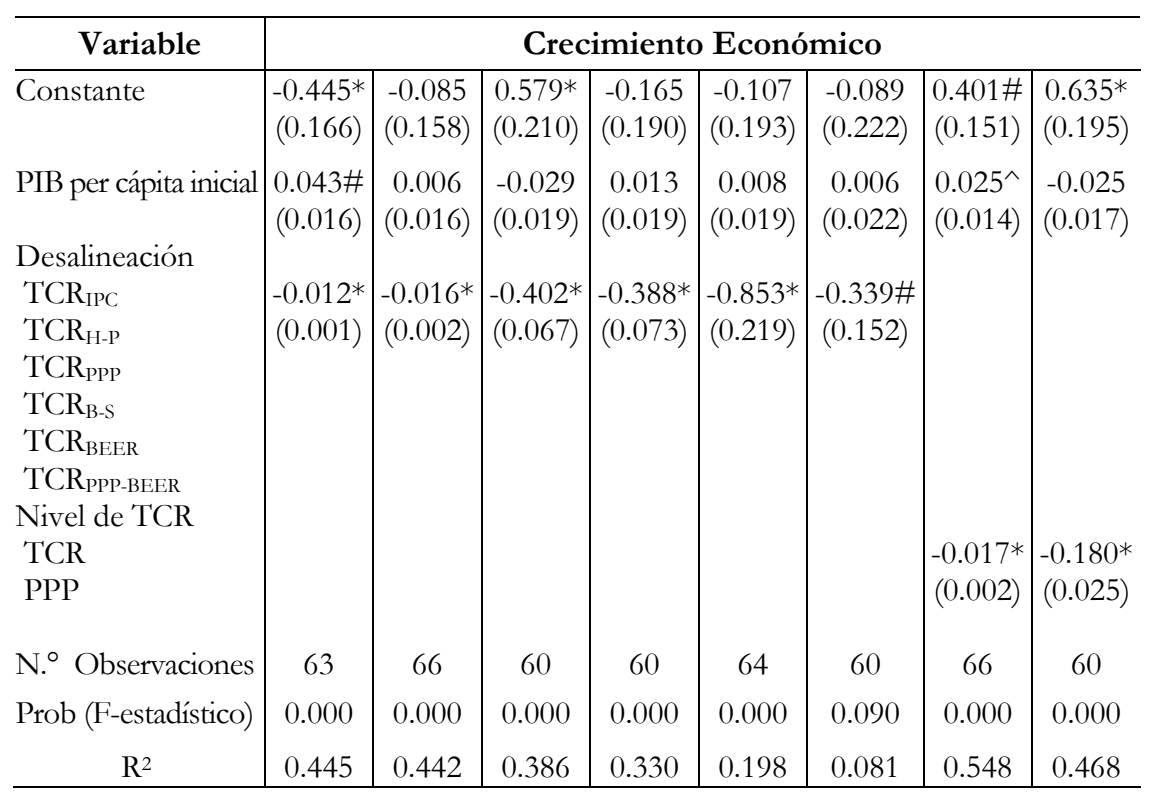

Fuente: Elaboración propia.

Nota: Los errores estándar están en paréntesis. Los símbolos *, \# y ^ representan un nivel de significancia de $1 \%, 5 \%$ y $10 \%$, respectivamente.

$\mathrm{Si}$ nos guiamos por el criterio del $\mathrm{R}^{2}$ vemos que las especificaciones tienden a explicar un bajo porcentaje de la variabilidad de la tasa de crecimiento, pero estos resultados son consistentes con otros estudios empíricos sobre crecimiento. ${ }^{13} \mathrm{El}$ coeficiente de la variable desalineamiento es significativo al $1 \%$ en la mayoría de las especificaciones y muestra una relación negativa con el crecimiento económico en todas las estimaciones, lo que indica que cuando se incrementa el ingreso per cápita, el efecto de una depreciación

13 Véase Bastourre, Casanova y Espora, 2011. 
del tipo de cambio real sobre el crecimiento es menor, en otras palabras, el crecimiento económico causa una apreciación real. En otro orden, lo que muestran los resultados es que un tipo de cambio más "competitivo" tiene un efecto negativo sobre el crecimiento. Es decir, una depreciación del tipo de cambio real provoca una disminución del crecimiento. En las dos primeras columnas, aunque el coeficiente es estadísticamente significativo, la magnitud del mismo implica que su importancia en términos económicos es leve, por lo que se requeriría una alta desalineación para generar un efecto importante en el crecimiento de la República Dominicana. En cambio, los resultados de las columnas 3, 4, 5 y 6 muestran coeficientes estadísticamente significativos y con una magnitud importante. Asimismo, cuando se evalúa el efecto del nivel del TCR sobre el crecimiento, podemos observar que los coeficientes muestran un efecto negativo y estadísticamente significativo, siendo de mayor magnitud cuando se utiliza el tipo de cambio real corregido por la paridad del poder adquisitivo. Estos resultados son congruentes con los de Sánchez-Fung (2005b), quien muestra que las depreciaciones generan un impacto negativo sobre el producto. En cuanto al ingreso inicial, dicha variable tiene el signo esperado en dos de las estimaciones realizadas, pero no es significativo estadísticamente. Una vez concluido esta parte se procedió a estimar la ecuación (6). Los resultados se encuentran en la tabla N. ${ }^{\circ} 4$. En dicha tabla se reportan los coeficientes individuales de la desalineación actual y su efecto de largo plazo, el cual conlleva a la realización del test de Wald para probar la significancia conjunta, $\sum_{i=0}^{1} \hat{\delta}_{i}=0$.

Los coeficientes de la desalineación rezagada en un período son significativos al 1\% en cinco de las seis regresiones. En las dos primeras columnas, la desalineación o subvaluación rezagada permanecen estables en un rango de 0.009 y 0.010 , mientras que en las tres siguientes están en un rango entre 0.561 y 0.986 . Lo anterior podría sugerir que en algunas ocasiones se hace necesario un tipo de cambio más competitivo para estimular el crecimiento económico. 
Sin embargo, el efecto actual de la desalineación (subvaluación) del tipo de cambio real es negativo y significativo al $1 \%$ en las primeras cinco estimaciones. En las dos primeras columnas, la desalineación es estable en torno a -0.011 , y en las tres siguientes se mantiene en un rango entre -0.943 y -1.020 . En la columna 6, los coeficientes de la desalineación son negativos en ambos casos. En otro orden, el efecto positivo de la desalineación rezagada en la columna 2 parece ser neutralizado por el efecto negativo de la desalineación en el período actual. Estos resultados de cambios de signos entre la desalineación rezaga y la del período actual son similares a los encontrados por Razmi, Rappetti y Skott (2012). Asimismo, cuando consideramos el efecto permanente o de largo plazo de la desalineación o subvaluación del tipo de cambio real sobre el crecimiento económico, se observa una tendencia a ser pequeño y negativo en las columnas 1 y 3 , y medianamente alto en las columnas 5 y 6 . En la columna 4, dicho efecto se presenta positivo, pero solo son significativo las columnas 1, 3, 4 y 5 de acuerdo al test de Wald que indica significancia al 1\% para los resultados mostrados en las columnas 3,4 y 5 , y al $5 \%$ en la columna 1 .

Los resultados evidenciados en la tabla N. ${ }^{\circ} 4$ sugieren que el crecimiento económico mostrado por la República Dominicana ha estado influenciado, básicamente, por la acumulación de capital físico medido como el ratio inversión a PIB y el tamaño de la población. Los coeficientes de ambas variables se muestran positivos y significativos. La relación positiva entre la población y el crecimiento económico es consistente con los resultados encontrados por Pozo, Sánchez-Fung y Santos-Paulino (2011) para la economía dominicana. En cambio, tanto la acumulación de capital humano (educación) como la apertura económica, no muestran el signo esperado. Esta última variable, solo es significativa en las columnas 5 y 6 . En cuanto al capital humano, medido como escolaridad, el signo negativo es consistente con los resultados para economías de los países del oriente medio y el norte de África (Los llamados países MENA, por sus siglas en inglés). El 
nivel inicial de esta variable podría explicar su signo. Además es consistente con los resultados de Barro (1999), quien muestra una contribución negativa de dicha variable al crecimiento económico de la República Dominicana.

En cuanto al grado de apertura de la economía, dicha variable solo muestra el signo esperado (positivo) en las primeras dos regresiones, pero no son estadísticamente significativas. En cambio, en las cuatro restantes muestra un signo negativo, siendo estadísticamente significativo en las dos últimas. Una posible razón para estos resultados puede ser que la muestra abarca una cantidad importante de años en la que habían tenido lugar políticas de sustitución de importaciones. Por consiguiente, cuanto más exitoso fue el sesgo contra las exportaciones, más altas fueron las tasas de crecimiento en las primeras etapas de la sustitución de importaciones. El signo negativo mostrado por la apertura económica es consistente con los resultados exhibidos por Pozo, Sánchez-Fung y Santos-Paulino (2011). Por su parte, los términos de intercambio tienen signo negativo pero no resultó significativo en ninguna de las regresiones, un resultado similar a los encontrados por Gregorio (1992) y Razmi, Rappetti y Skott (2012). Una posible explicación para estos resultados reside en el hecho de que la economía dominicana depende, en gran medida, de la importación de petróleo y es sumamente vulnerable al medio ambiente externo; por tanto, los resultados podrían estar reflejando un deterioro de los términos de intercambio. Con respecto a la inflación, la misma tiene el signo esperado y es estadísticamente significativa en todas las estimaciones, una alta tasa de inflación está correlacionada con un crecimiento económico más bajo. De manera más específica, la estimación del coeficiente implica que una tasa de inflación de 10\% anual está asociada con un nulo crecimiento per cápita. 
Tabla N. ${ }^{\circ} 4$

Estimación variables instrumentatales del crecimiento

\begin{tabular}{|c|c|c|c|c|c|c|}
\hline \multirow{2}{*}{$\frac{\text { Variable }}{\text { Constante }}$} & \multicolumn{6}{|c|}{ Crecimiento Económico } \\
\hline & $\begin{array}{l}1.242^{*} \\
(0.423)\end{array}$ & $\begin{array}{l}0.981^{\wedge} \\
(0.575)\end{array}$ & $\begin{array}{c}0.952 \\
(0.648)\end{array}$ & $\begin{array}{c}1.384 \\
(0.828)\end{array}$ & $\begin{array}{c}0.811 \\
(0.753)\end{array}$ & $\begin{array}{l}1.599 \\
(1.086)\end{array}$ \\
\hline PIB per cápita inicial & $\begin{array}{c}-0.050 \# \\
(0.023)\end{array}$ & $\begin{array}{l}-0.041 \\
(0.033)\end{array}$ & $\begin{array}{l}-0.030 \\
(0.037)\end{array}$ & $\begin{array}{l}-0.053 \\
(0.048)\end{array}$ & $\begin{array}{l}-0.022 \\
(0.043)\end{array}$ & $\begin{array}{l}-0.070 \\
(0.060)\end{array}$ \\
\hline Desalineación & & & & & & \\
\hline $\begin{array}{l}\text { TCR }_{\text {IPC }} \\
\text { TCR }_{\text {IPC } t-1}\end{array}$ & $\begin{array}{c}-0.011^{*} \\
(0.001)\end{array}$ & $\begin{array}{l}-0.011 * \\
(0.001)\end{array}$ & $\begin{array}{l}-0.963^{*} \\
(0.135)\end{array}$ & $\begin{array}{c}-0.943^{*} \\
(0.167)\end{array}$ & $\begin{array}{l}-1.020^{*} \\
(0.184)\end{array}$ & $\begin{array}{l}-0.093 \\
(0.210)\end{array}$ \\
\hline $\mathrm{TCR}_{\mathrm{H}-\mathrm{Pt}}$ & $0.009 *$ & $0.010^{*}$ & $0.955^{*}$ & $0.986^{*}$ & $0.561^{*}$ & -0.127 \\
\hline 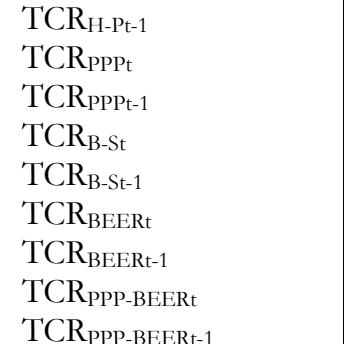 & $(0.001)$ & $(0.001)$ & $(0.116)$ & $(0.141)$ & $(0.188)$ & $(0.171)$ \\
\hline Educación & $\begin{array}{l}-1.514 * \\
(0.369)\end{array}$ & $\begin{array}{l}-0.337 \\
(0.396)\end{array}$ & $\begin{array}{l}-1.482^{*} \\
(0.536)\end{array}$ & $\begin{array}{c}-1.819 * \\
(0.584)\end{array}$ & $\begin{array}{c}-0.972^{\wedge} \\
(0.564)\end{array}$ & $\begin{array}{l}-2.305^{*} \\
(0.828)\end{array}$ \\
\hline Apertura & $\begin{array}{l}0.0793 \\
(0.086)\end{array}$ & $\begin{array}{c}0.114 \\
(0.118)\end{array}$ & $\begin{array}{l}-0.027 \\
(0.126)\end{array}$ & $\begin{array}{l}-0.082 \\
(0.139)\end{array}$ & $\begin{array}{l}-0.546^{*} \\
(0.151)\end{array}$ & $\begin{array}{c}-0.443 \# \\
(0.197)\end{array}$ \\
\hline Inflación & $\begin{array}{c}-0.992 * \\
(0.098)\end{array}$ & $\begin{array}{l}-1.00^{*} \\
(0.134)\end{array}$ & $\begin{array}{l}-0.860^{*} \\
(0.127)\end{array}$ & $\begin{array}{c}-0.943^{*} \\
(0.143)\end{array}$ & $\begin{array}{c}-0.399 \# \\
(0.156)\end{array}$ & $\begin{array}{c}-0.430 \# \\
(0.178)\end{array}$ \\
\hline Población & $\begin{array}{l}1.242^{*} \\
(0.296)\end{array}$ & $\begin{array}{c}0.129 \\
(0.298)\end{array}$ & $\begin{array}{l}1.261 * \\
(0.415)\end{array}$ & $\begin{array}{l}1.509 * \\
(0.453)\end{array}$ & $\begin{array}{l}0.766^{\wedge} \\
(0.438)\end{array}$ & $\begin{array}{l}1.918^{*} \\
(0.640)\end{array}$ \\
\hline Consumo del gobierno & $\begin{array}{l}-0.713^{\wedge} \\
(0.365)\end{array}$ & $\begin{array}{c}-1.082 \# \\
(0.466)\end{array}$ & $\begin{array}{l}-0.566 \\
(0.485)\end{array}$ & $\begin{array}{l}-0.758 \\
(0.535)\end{array}$ & $\begin{array}{c}-1.412 \# \\
(0.438)\end{array}$ & $\begin{array}{l}-1.508^{\wedge} \\
(0.791)\end{array}$ \\
\hline Inversión & $\begin{array}{l}0.177^{*} \\
(0.055)\end{array}$ & $\begin{array}{c}0.017 \\
(0.067)\end{array}$ & $\begin{array}{l}0.209 \# \\
(0.085)\end{array}$ & $\begin{array}{l}0.270^{*} \\
(0.093)\end{array}$ & $\begin{array}{l}0.358^{*} \\
(0.087)\end{array}$ & $\begin{array}{l}0.404^{*} \\
(0.112)\end{array}$ \\
\hline Términos de intercambio & $\begin{array}{l}-0.116 \\
(0.091)\end{array}$ & $\begin{array}{l}-0.008 \\
(0.124)\end{array}$ & $\begin{array}{l}-0.136 \\
(0.127)\end{array}$ & $\begin{array}{l}-0.179 \\
(0.140)\end{array}$ & $\begin{array}{l}-0.024 \\
(0.160)\end{array}$ & $\begin{array}{l}-0.171 \\
(0.201)\end{array}$ \\
\hline $\begin{array}{l}\text { LnDestcr }{ }_{\mathrm{t}}+\text { LnDestcr }_{\mathrm{t}-1} \\
\text { Test de Wald }\end{array}$ & -0.002 & -0.000 & -0.007 & 0.043 & -0.458 & -0.220 \\
\hline $\begin{array}{l}\chi^{2} \\
\text { p-value }\end{array}$ & $\begin{array}{c}6.775 \\
(0.012)\end{array}$ & $\begin{array}{c}2.355 \\
(0.130)\end{array}$ & $\begin{array}{l}65.021 \\
(0.000)\end{array}$ & $\begin{array}{l}45.493 \\
(0.000)\end{array}$ & $\begin{array}{l}33.667 \\
(0.000)\end{array}$ & $\begin{array}{c}0.755 \\
(0.384)\end{array}$ \\
\hline N. ${ }^{\circ}$ Observaciones & 62 & 64 & 59 & 59 & 63 & 59 \\
\hline Prob (F-estadístico) & 0.000 & 0.000 & 0.000 & 0.000 & 0.000 & 0.000 \\
\hline $\mathrm{R}^{2}$ & 0.908 & 0.833 & 0.841 & 0.804 & 0.699 & 0.619 \\
\hline
\end{tabular}

Fuente: Elaboración propia.

Nota: Los errores estándar están en paréntesis. Los símbolos *, \# y ^ representan un nivel de significancia de $1 \%, 5 \%$ y $10 \%$, respectivamente. 
Crecimiento económico y desalineación del tipo de cambio real en la República Dominicana: ¿Hay alguna relación?

Tabla N. ${ }^{\circ} 5$

Estimación de la depreciación del TCR y el crecimiento

\begin{tabular}{|c|c|c|c|c|c|c|}
\hline \multirow{2}{*}{$\frac{\text { Variable }}{\text { Constante }}$} & \multicolumn{6}{|c|}{ Crecimiento Económico } \\
\hline & $\begin{array}{l}1.524^{\wedge} \\
(0.882)\end{array}$ & $\begin{array}{l}1.564^{\wedge} \\
(0.885)\end{array}$ & $\begin{array}{c}1.106 \\
90.934)\end{array}$ & $\begin{array}{l}1.686^{\wedge} \\
(0.965)\end{array}$ & $\begin{array}{c}0.654 \\
(0.888)\end{array}$ & $\begin{array}{l}1.862 \# \\
(0.896)\end{array}$ \\
\hline PIB per cápita inicial & $\begin{array}{l}-0.068 \\
(0.050)\end{array}$ & $\begin{array}{l}-0.070 \\
(0.050)\end{array}$ & $\begin{array}{l}-0.048 \\
(0.052)\end{array}$ & $\begin{array}{l}-0.076 \\
(0.058)\end{array}$ & $\begin{array}{l}-0.014 \\
(0.051)\end{array}$ & $\begin{array}{l}-0.082 \\
(0.050)\end{array}$ \\
\hline \multicolumn{7}{|l|}{ Depreciación } \\
\hline DummyipCt & $-0.078 \#$ & $-0.061^{\wedge}$ & $-0.148 \#$ & -0.002 & $-0.094 *$ & -0.055 \\
\hline Dummyн-Pt & $(0.036)$ & $(0.032)$ & $(0.065)$ & $(0.060)$ & $(0.033)$ & $(0.033)$ \\
\hline \multicolumn{7}{|l|}{ DummyppPt } \\
\hline \multicolumn{7}{|l|}{ DummyB-St } \\
\hline \multicolumn{7}{|l|}{ DummybeERt } \\
\hline \multicolumn{7}{|l|}{ Dummypp-BEERt } \\
\hline \multirow[t]{2}{*}{ Educación } & -0.986 & -0.877 & -0.743 & $-1.322^{\wedge}$ & -0.373 & $-2.514^{*}$ \\
\hline & $(0.691)$ & $(0.615)$ & $(0.733)$ & $(0.723)$ & $(0.181)$ & $(0.736)$ \\
\hline \multirow[t]{2}{*}{ Apertura } & $-0.317^{\wedge}$ & -0.284 & -0.218 & $-0.327^{\wedge}$ & $-0.547^{*}$ & $-0.385 \#$ \\
\hline & $(0.172)$ & $(0.178)$ & $(0.186)$ & $(0.190)$ & $(0.181)$ & $(0.165)$ \\
\hline \multirow[t]{2}{*}{ Inflación } & $-0.495^{*}$ & $-0.626^{*}$ & $-0.385^{\wedge}$ & $-0.575^{*}$ & $-0.402 \#$ & $-0.468^{*}$ \\
\hline & $(0.176)$ & $(0.178)$ & $(0.194)$ & $(0.189)$ & $(0.181)$ & $(0.165)$ \\
\hline \multirow[t]{2}{*}{ Población } & 0.664 & 0.519 & 0.524 & $0.953^{\wedge}$ & 0.191 & $2.061^{*}$ \\
\hline & $(0.541)$ & $(0.468)$ & $(0.567)$ & $(0.563)$ & $(0.466)$ & $(0.591)$ \\
\hline \multirow[t]{2}{*}{ Consumo del gobierno } & $-1.351^{\wedge}$ & $-1.931 \#$ & -1.191 & $-1.766 \#$ & $-1.722 \#$ & $-1.551 \#$ \\
\hline & $(0.755)$ & $(0.755)$ & $(0.786)$ & $(0.854)$ & $(0.723)$ & $(0.723)$ \\
\hline \multirow[t]{2}{*}{ Inversión } & $0.216 \#$ & 0.173 & 0.178 & $0.265 \#$ & $0.247 \#$ & $0.390^{*}$ \\
\hline & $(0.104)$ & $(0.108)$ & $(0.111)$ & $(0.111)$ & $(0.099)$ & $(0.106)$ \\
\hline \multirow[t]{2}{*}{ Términos de Intercambio } & -0.167 & -0.076 & -0.098 & -0.138 & 0.031 & -0.201 \\
\hline & $(0.190)$ & $(0.192)$ & $(0.194)$ & $(0.203)$ & $(0.188)$ & $(0.185)$ \\
\hline N. ${ }^{\circ}$ Observaciones & 63 & 64 & 60 & 60 & 64 & 60 \\
\hline Prob (F-estadístico) & 0.000 & 0.000 & 0.000 & 0.000 & 0.000 & 0.000 \\
\hline $\mathrm{R}^{2}$ & 0.561 & 0.549 & 0.569 & 0.525 & 0.582 & 0.623 \\
\hline
\end{tabular}

Fuente: Elaboración propia.

Nota: Los errores estándar están en paréntesis. Los símbolos *, \# y ^ representan un nivel de significancia de $1 \%, 5 \%$ y $10 \%$, respectivamente.

Con respecto al consumo de gobierno, dicha variable tiene signos negativos y es estadísticamente significativa en la mayoría de las regresiones, lo que implica que el gasto del gobierno dominicano ha afectado de manera negativa el crecimiento económico. Este resultado es congruente con los obtenidos por Barro (1991), Edwards 
(1992), Calderón y Fuentes (2006), Jaramillo y Sancak (2007) y McLoed y Mileva (2011). Asimismo, el ingreso per cápita muestra el signo correcto pero solo es significativo en la primera regresión, lo cual no es prueba de que exista una convergencia (incondicional) del PIB. Este último resultado es congruente con los mostrados por De Gregorio (1992) para los países de América Latina. En términos generales, en cada una de estas regresiones los $\mathrm{R}^{2}$ son altos, lo que implica que el modelo empírico es capaz de explicar más de las tres cuartas partes de la variabilidad del crecimiento promedio de la República Dominicana.

Una limitante de los resultados anteriores es que no nos permiten evaluar si la sobrevaluación o la subvaluación tienen efectos asimétricos sobre el crecimiento. Por tanto, se estima la ecuación (7). Para ello, primero se discuten los resultados de la subvaluación (depreciación) mostrados en la tabla N. ${ }^{\circ}$ 5. Luego se discuten los resultados de la sobrevaluación (apreciación) mostrados en la tabla N. ${ }^{\circ}$ 6. Como puede observarse en la tabla N. ${ }^{\circ} 5$, todos los coeficientes de la variable Dummy tienen signos negativos, aunque no todos son estadísticamente significativos. Económicamente hablando, cuando se producen depreciaciones del TCR se reduce el crecimiento de la República Dominicana.

Asimismo, la variable educación o capital humano muestra un signo negativo en todas las regresiones, pero solo es estadísticamente significativa en dos. La apertura económica muestra una relación negativa con el crecimiento económico en todas las regresiones, siendo estadísticamente significativa en cuatro de ellas. ${ }^{14}$ De igual

14 Además, Peguero y Cruz-Rodríguez (2015) señalan que ante procesos de devaluaciones o depreciaciones del tipo de cambio nominal en la economía dominicana, las autoridades intervienen rápidamente dada la sensibilidad política de la variable cambiaria. En consecuencia, la curva J no opera correspondientemente y los beneficios en términos de exportaciones no se perciben. La mejora de la balanza comercial es solo por monto, al convertir dólares a pesos, pero no por incremento del volumen exportado. De igual forma, Sánchez-Fung (2005a) identifica una especie "miedo a dejar flotar" el tipo de cambio en la economía dominicana. 
forma, los efectos negativos de la inflación contra el crecimiento parecen ser muy importantes en todas las especificaciones. En ese sentido, De Gregorio (1993) presenta un modelo de crecimiento endógeno en el cual la inflación es el resultado de un sistema tributario ineficiente, por tanto, cuando se requiere dinero para comprar bienes de capital la inflación es similar a un impuesto sobre la inversión. Además, dicho autor muestra cómo la inflación puede afectar la productividad del capital. Desde el punto de vista de dicho autor, la inflación induce a la gente y a las empresas a desviar recursos de las actividades productivas para destinarlos a otras actividades que les permitan reducir la carga del impuesto inflación, afectando negativamente al crecimiento.

En otro orden, la variable población continúa mostrando su relación positiva con el crecimiento económico aunque solo es significativa en dos regresiones. Los incrementos del consumo de gobierno continúan afectando negativamente el crecimiento de la actividad económica, siendo estadísticamente significativo en la mayoría de los resultados. Por su parte, la inversión continúa mostrando una relación positiva con el crecimiento, siendo estadísticamente significativa en la mayoría de las regresiones, mientras que los términos de intercambios muestran una relación negativa, pero no son estadísticamente significativos en ninguno de los resultados. De forma similar, el PIB inicial muestra el signo correcto pero no es significativo en ninguna de las regresiones. En cuanto al ajuste de las regresiones, los $\mathrm{R}^{2}$ son superiores al $50 \%$ en todos los resultados, lo que implica que el modelo empírico es capaz de explicar más de la mitad de la variabilidad del crecimiento promedio de la República Dominicana. 
Tabla N. ${ }^{\circ} 6$

Estimación de la apreciación del TCR y el crecimiento

\begin{tabular}{|c|c|c|c|c|c|c|}
\hline Variable & & $\mathrm{Cr}$ & ecimient & Econón & ico & \\
\hline Constante & 1.446 & $1.502^{\wedge}$ & 0.958 & $1.684^{\wedge}$ & 0.560 & $1.808^{\wedge}$ \\
\hline & $(0.886)$ & $(0.883)$ & $(0.954)$ & $(0.980)$ & $(0.899)$ & $(0.908)$ \\
\hline PIB per cápita inicial & -0.068 & -0.070 & -0.049 & -0.077 & -0.015 & -0.083 \\
\hline & $(0.050)$ & $(0.050)$ & $(0.065)$ & $(0.058)$ & $(0.051)$ & $(0.051)$ \\
\hline Apreciación & & & & & & \\
\hline DummyIPCt & $0.078 \#$ & $0.061^{\wedge}$ & $0.148 \#$ & 0.002 & $0.094^{*}$ & 0.055 \\
\hline DummyH-Pt & $(0.036)$ & $(0.032)$ & $(0.065)$ & $(0.064)$ & $(0.033)$ & $(0.034)$ \\
\hline DummyppPt & & & & & & \\
\hline Dummyв-St & & & & & & \\
\hline DummybeERt & & & & & & \\
\hline DummyPP-BEERt & & & & & & \\
\hline Educación & -0.986 & -0.887 & -0.744 & $-1.323^{\wedge}$ & -0.374 & $-2.514^{*}$ \\
\hline & $(0.691)$ & $(0.615)$ & $(0.733)$ & $(0.723)$ & $(0.614)$ & 0.737 \\
\hline Apertura & $-0.317^{\wedge}$ & -0.284 & -0.219 & $-0.327^{\wedge}$ & $-0.548^{*}$ & $-0.386 \#$ \\
\hline & $(0.172)$ & $(0.178)$ & $(0.186)$ & $(0.191)$ & $(0.182)$ & $(0.166)$ \\
\hline Inflación & $-0.495^{*}$ & $-0.626^{*}$ & $-0.386^{\wedge}$ & $-0.576^{*}$ & $-0.402 \#$ & $-0.469 *$ \\
\hline & $(0.176)$ & $(0.178)$ & $(0.195)$ & $(0.189)$ & $(0.181)$ & $(0.165)$ \\
\hline Población & 0.664 & 0.519 & 0.524 & $0.954^{\wedge}$ & 0.191 & $2.061^{*}$ \\
\hline & $(0.541)$ & $(0.468)$ & $(0.567)$ & $(0.563)$ & $(0.466)$ & $(0.591)$ \\
\hline Consumo del gobierno & $-1.351^{\wedge}$ & $-1.931 \#$ & -1.191 & $-1.767 \#$ & $-1.722 \#$ & $-1.551 \#$ \\
\hline & $0.755)$ & $(0.755)$ & $(0.786)$ & $(0.855)$ & $(0.723)$ & $(0.723)$ \\
\hline Inversión & $0.216 \#$ & 0.173 & 0.178 & $0.265 \#$ & $0.247 \#$ & $0.391 *$ \\
\hline & $(0.104)$ & $(0.1080$ & $(0.111)$ & $(0.111)$ & $(0.099)$ & $(0.106)$ \\
\hline Términos de Intercambio & -0.167 & -0.076 & -0.099 & -0.138 & 0.031 & -0.202 \\
\hline & $(0.190)$ & $(0.192)$ & (0.194) & $(0.203)$ & $(0.1889)$ & $(0.185)$ \\
\hline N. ${ }^{\circ}$ Observaciones & 63 & 64 & 60 & 60 & 64 & 60 \\
\hline Prob (F-estadístico) & 0.000 & 0.000 & 0.000 & 0.000 & 0.000 & 0.000 \\
\hline $\mathrm{R}^{2}$ & 0.561 & 0.549 & 0.570 & 0.525 & 0.582 & 0.623 \\
\hline
\end{tabular}

Fuente: Elaboración propia.

Nota: Los errores estándar están en paréntesis. Los símbolos * \# $\mathrm{y}^{\wedge}$ representan un nivel de significancia de $1 \%, 5 \%$ y $10 \%$, respectivamente.

Por otro lado, la tabla N. ${ }^{\circ} 6$ muestra los resultados de la sobrevaluación (apreciación) del TCR. En ella se observa el efecto contrario a la tabla N. ${ }^{\circ}$, todos los coeficientes de la variable Dummy tienen signos positivos, aunque no todos son estadísticamente significativos. Estos resultados estarían en línea con la visión del Consenso de Washington de que el desarrollo económico exitoso resulta en 
una apreciación de la tasa de cambio real y en un mejoramiento del nivel de vida. No obstante, estaríamos entrando en una relación de causalidad, de si la apreciación del TCR causa crecimiento económico o el crecimiento económico causa una apreciación del tipo de cambio real. Sin embargo, eso traspasa el límite de esta investigación.

En general, los resultados mostrados en la tabla N. ${ }^{\circ} 6$ son similares a los presentados en la tabla $N .^{\circ} 5$. El capital humano, el grado de apertura de la economía, la inflación, el consumo del gobierno y los términos de intercambio tienen un efecto negativo sobre el crecimiento, aunque no todos son estadísticamente significativos. Mientras que la población y la acumulación de capital físico tienen un efecto positivo. Asimismo, el ingreso per capita inicial muestra el signo correcto en todas las regresiones pero no es significativo en ninguna de ellas. Al igual que los resultados de la tabla $\mathrm{N} .^{\circ} 5$, los $\mathrm{R}^{2}$ mostrados en la tabla 6 son superiores al 50\% en todos los resultados, lo que indica un buen ajuste para explicar la variabilidad del crecimiento promedio de la República Dominicana.

\section{Conclusión}

En este artículo se aborda la cuestión de que si la desalineación (depreciación o apreciación) del tipo de cambio real afecta el crecimiento económico de la República Dominicana. Los resultados sugieren que el crecimiento es adversamente afectado por la depreciación del tipo de cambio real. Un tipo de cambio real más "competitivo" para incentivar las inversiones orientadas a la exportación y el crecimiento económico, que implica un proceso de devaluación o depreciación del tipo de cambio nominal, no conduce al crecimiento económico en el mediano y largo plazo. El efecto positivo de una depreciación del tipo de cambio real, encontrado en nuestras estimaciones, opera principalmente a través del primer rezago, pero su coeficiente es muy bajo. Además, una política de 
orientación hacia fuera requiere algún tiempo para producir beneficios y en la República Dominicana el tema cambiario es muy sensible a las decisiones de las autoridades políticas. Niveles muy altos de tipo de cambio real están asociados a crisis cambiarias más que a un deseo de las autoridades de tener una política cambiaria más competitiva, en ese sentido, las depreciaciones en el tipo de cambio real pueden no redundar en la apertura de nuevos mercados e incremento de los productos de exportación, dado que los agentes perciben los altos valores en el tipo de cambio real como transitorios. Esto es consecuente con lo señalado por Montiel (2006) quien sostiene que el país necesita una política de tipo de cambio nominal que garantice que el tipo de cambio real de equilibrio resultante no sea oscurecido por episodios repetidos de sobrevaluación o excesiva inestabilidad a corto plazo. Dicha política puede influir en el tipo de cambio real de equilibrio que impulsa las decisiones de asignación de recursos intersectorial a largo plazo.

Con respecto a las otras variables incluidas como determinantes del crecimiento, la acumulación de capital físico, medido como el ratio inversión a PIB, ha tenido una alta influencia positiva en el crecimiento económico de la República Dominicana. De igual forma, la población tiene un efecto positivo sobre la expansión de la actividad económica. En cambio, el capital humano muestra una relación negativa con el mismo. No obstante, las inversiones en la calidad de la educación podrían hacer cambiar dicho signo. Asimismo, el grado de apertura de la economía, la inflación, el consumo del gobierno y los términos de intercambio tienen un efecto negativo sobre el crecimiento, aunque no todos son estadísticamente significativos. Los efectos negativos de la inflación contra el crecimiento parecen ser muy importantes en todas las especificaciones utilizadas en el presente documento. Asimismo, no encontramos evidencias de convergencia del ingreso per cápita a pesar de que dicha variable muestra el signo correcto. 
Para futuras investigaciones se deben explorar canales alternativos por los cuales un tipo de cambio real sobrevaluado opera sobre el crecimiento, esto podría explicar las razones subyacentes que se manifiestan en los resultados expresados en este trabajo.

\section{Referencias bibliográficas}

Abida, Z. (2011). Real exchange rate misalignment and economic growth: An empirical study for the Maghreb countries. International Journal of Economics and Finance, 3(3), 190-201.

Aguirre, A. \& Calderón, C. (2006). Real exchange rate misalignments and economic performance. Working Paper N. ${ }^{\circ} 315$, Banco Central de Chile.

Balassa, B. (1964). The purchasing power parity doctrine: A reappraisal. Journal of Political Economy, 72, 584-596.

Barro, R. (1991). Economic growth in a cross-section countries. The Quarterly Journal of Economics, 106(2), 407-443.

Barro, R. (1999). Determinats of economic growth: Implications of the global evidence for Chile. Cuadernos de Economia, 36(107), 443-478.

Barro, R. \& Sala-i-Martin, X. (1990). Economic growth and convergence across the United States. Working Paper N. ${ }^{\circ}$ 3419. National Bureau of Economic Research.

Bastourre, D.; Casanova, L. \& Espora, A. (2011). Tipo de cambio real y crecimiento: Sintesis de la evidencia y agenda de investigación. (Documento de trabajo N. ${ }^{\circ}$ 82). Buenos Aires: Universidad Nacional de La Plata.

Beréau, S.; López Villavicencio, A. \& Mignon, V. (2012). Currency misalignments and growth: A new look using nonlinear panel data methods. Applied Economics, 44(27), 3503-3511. 
Berg, A. \& Miao, Y. (2010). The Real Exchange Rate and Growth Revisited: The Washington Consensus Strikes Back? Working Paper WP/10/58, International Monetary Fund.

Bussiere, M.; López, C. \& Tille, C. (2014). Do real Exchange rate appreciations matter for growth? Working Paper 06/2014. Graduate Institute of International and Development Studies.

Calderón, C. \& Fuentes, R. (2006). ¿Cuánto explican las reformas y la calidad de las instituciones del crecimiento chileno?: Una comparación internacional. Monetaria, 29(2), 119-149.

Cottani, J.; Cavallo, D. F. \& Khan, M. S. (1990). Real Exchange rate behavior and economic performance in LDCs. Economic Development and Cultural Change, 39(1), 61-76.

Cruz-Rodríguez, A. (2015). Tipo de cambio real en la República Dominicana: Enfoques alternativos de equilibrio y desalineamiento. [Mimeo].

Debowicz, D. \& Saeed, W. (2014). Exchange rate misalignment and economic development: The case of Pakistan. [Working Paper 210]. Brooks World Poverty Institute. The University of Manchester.

Domac, I., \& Shabsigh, G. (1999). Real exchange rate behavior and economic growth: Evidence from Egypt, Jordan, Morocco, and Tunisia. Working Paper WP/99/40, International Monetary Fund.

Edwards, S. (1989). Exchange rate misalignment in developing countries. The World Bank Research Observer, 4(1), 3-21.

Edwards, S. (1992). Orientación del comercio exterior, deformaciones y crecimiento en los países en desarrollo. El Trimestre Económico, 59, 41-74. 
Edwards, S. \& Savastano, M. (1999). Exchange rates in Emerging Economies: What do we know? What do we need to know? Working Paper N. ${ }^{\circ}$ 7228. National Bureau of Economic Research.

Eichengreen, B. (2008). The real exchange rate and economic growth. Commission on Growth and Development, Working Paper N. ${ }^{\circ}$. The World Bank.

Fanelli, J. M. \& Guzmán, R. (2008). Diagnóstico de crecimiento para la República Dominicana. (Documento de Trabajo CSI-118). Banco Interamericano de Desarrollo.

Gala, P. \& Lucinda, C. R. (2006). Exchange Rate Misalignment and Growth: Old and New Econometric Evidence. Revista Economía, 7(8), 165-187.

Gala, P. (2008). Real exchange rate levels and economic development: Theoretical analysis and econometric evidence. Cambridge Journal of Economics, 32, 273-288.

Gregorio, J. De. (1992). El crecimiento económico en América Latina. El Trimestre Económico, 59, 75-107.

Gregorio, J. De. (1993). Inflation, taxation and long-run growth. Journal of Monetary Economics, 31(3), 271-298.

Griffin, N. N. (2015). Determinants of firm profitability in Colombia's manufacturing sector: Exchange rate or structural? Working Paper WP/15/97. International Monetary Fund.

Harberger, A. C. (2003). Economic growth and the real exchange rate: Revisiting the Balassa-Samuelson effect. Moscow: The Higher School of Economics.

Henrique Vaz, P. \& Baer, W. (2014). Real Exchange rate and manufacturing growth in Latin America. Latin American Economic Review, 23(2), 1-17. 
Hodrick, R. T. \& Prescott, E. C. (1997). Postwar US business cycles: An empirical investigation. Journal of Money, Credit and Banking. 29(1), 1-16.

Ito, T.; Isard, P. \& Symansky, S. (1999). Economic growth and real exchange rate: An overview of the Balassa-Samuelson hypothesis in Asia. En T. Ito \& A. O. Krueger (Eds.). Changes in exchange rates in rapidly development countries: Theory, practice and policy issues. Chicago: University of Chicago Press.

Jaramillo, L. \& Sancak, C. (2007). Growth in the Dominican Republic and Haiti: Why has the grass been greener on one side of Hispaniola? Working Paper WP/07/63. International Monetary Fund.

Lucas, R. E. (1988). On the mechanics of economic development. Journal of Monetary Economics, 22, 3-42.

Mbaye, S. (2012). Real exchange rate undervaluation and growth: Is there a total factor productivity growth channel? (Etudes et Documents E 2012.11). Centre D'Etudes et de Recherches Sur le Developpment International.

McLoed, D. \& Mileva, E. (2011). Real exchange rates and productivity growth. Discussion Paper 2011-04. New York: Fordham University.

Montiel, P. J. (2006). Exchange rate policy and growth in the Dominican Republic. Massachusetts: Williams College.

Peguero, A. G. \& Cruz-Rodríguez, A. (2015). Condición MarshallLernery el efecto Curva J: Evidencias para la República Dominicana. [Mimeo].

Pozo, S.; Sánchez-Fung, J. R. \& Santos-Paulino, A. U. (2011). A note modelling economic growth determinants in the Dominican Republic. Macroeconomics and Finance in Emerging Economies, 4(1), 35-41. 
Rapetti, M.; Skott, P. \& Razmi, A. (2012). The real exchange rate and economic growth: Are developing countries different. International Review of Applied Economics, 26(6), 735-753.

Razin, O. \& Collins, S. M. (1997). Real Exchange Rate Misalignments and Growth. Working Paper N. ${ }^{\circ}$ 6174, National Bureau of Economic Research.

Razmi, A.; Rappetti, M. \& Skott, P. (2012). The real exchange rate and economic development. Structural Change and Economic Dynamics, 23, 151-169.

Ravn, M. \& Uhlig, H. (2002). On Adjusting the HP-Filter for the Frequency of Observations. The Review of Economics and Statistics, 84(2), 371-380.

Rodrik, D. (2008). The Real Exchange Rate and Economic Growth. Brookings Papers on Economic Activity, 2, 365-412.

Romer, P. M. (1986). Increasing returns and long-run growth. The Journal of Political Economy, 94(5), 1002-1037.

Samuelson, P. A. (1964). Theoretical notes on trade problems. Review of Economics and Statistics, 46, 145-164.

Sánchez-Fung, J. R. (2005a). Estimating a monetary policy reaction function for the Dominican Republic. International Economic Journal, 19(4), 563-577.

Sánchez-Fung, J. R. (2005b). Money demand, PPP, and macroeconomic dynamics in the Dominican Republic. Nordic Journal of Latin American and Caribbean Studies, 35(1), 61-80.

Solow, R. M. (1956). A contribution to the theory of economic growth. The Quarterly Journal of Economics, 70(1), 65-94.

Vieira, F. V. \& MacDonald, R. (2012). A panel data investigation of real exchange rate misalignment and growth. Estudios Económicos (Sao Paulo), 42(3), 433-456. 
Williamson, J. (1990). What Washington means by policy reform. En J. Williamson, J. (Ed.). Latin American Adjustment: How much has happened? Massachusetts: Peterson Institute for International Economics.

\section{Alexis Cruz Rodríguez}

Es doctor en Economía por la Universidad de Surrey, Inglaterra. Realizó un magíster en Economía Financiera en la Universidad de Santiago de Chile (USACH) y posee una licenciatura en Economía por el Instituto Tecnológico de Santo Domingo (INTEC). Actualmente se desempeña como director de la Oficina de Planificación y Desarrollo de la Superintendencia de Bancos de la República Dominicana y, concomitantemente, como profesor y coordinador de la Maestría en Economía Aplicada de la Pontificia Universidad Católica Madre y Maestra. Asimismo, se ha desempeñado como asesor de la Superintendencia de Bancos de la República Dominicana; coordinador del Área Macroeconómica del Ministerio de Economía, Planificación y Desarrollo; especialista Fiscal del Secretariado Técnico de la Presidencia; investigador Asociado de la Facultad Latinoamericana de Ciencias Sociales (FLACSO); ministro consejero de la Embajada Dominicana en el Reino Unido, Gran Bretaña e Irlanda del Norte; economista senior del Banco Estado, de Chile y de la Comisión Económica para América Latina y el Caribe (CEPAL). 

en la República Dominicana: ¿Hay alguna relación?

Ha impartido docencia en la Pontificia Universidad Católica Madre y Maestra, en la Universidad Católica Santo Domingo, en la Facultad Latinoamericana de Ciencias Sociales (FLACSO), en la Universidad de Surrey, la Universidad de Santiago de Chile, en la Universidad Católica Cardenal Silva Henríquez, en la Universidad Mayor y en el Instituto Tecnológico de Santo Domingo (INTEC). Sus trabajos académicos han sido publicados en revistas especializadas de circulación internacional tales como Theoretical and Applied Economics, International Journal of Economic Sciences and Applied Research, Review of Economic Perspectives, Revista de Análisis Económico, Revista Dominicana de Economía y en Ciencia y Sociedad, entre otras. De igual forma, ha sido árbitro de las revistas Journal of Economics and International Finance, Latin American Journal of Economics y Revista de Análisis Económico.

Recibido: 25/05/2015

Aprobado: 08/06/2015 
